Pneumonia, the result of the poison of influenza, has a much more anxious prognosis than the usual form of the disease. It is characterised by extreme asthenia, and often by copious perspiration; the range of temperature is not uniformly so high, and is far more irregular. From the rapid changes whici occur in the physical signs in influenzal pneumonia it may be conjectured that the pathological condition in the lungs is one of rapid engorgement or œdema.

The "crisis" is a prominent fact in the natural history of pneumonia. The date is postponed in direct relationship to the amount of lung tissue involved in the pathological process. Thus it occurs earlier with a limited than with an extensive lesion. The position is always an anxious one when the appearance of the crisis is delayed. The following may be mentioned as circumstances which interfere with a typical defervescence. Pericarditis, pleurisy, more especially of the purulent variety, the occurrence of pneumonia in the opposite lung, and bronchitis or catarrhal inflammation is not uncommon in children. In some cases influenzal pneumonia has a typical crisis, whilst in others no such event can be recognised. Particularly is it necessary to remember in cases of pneumonia where the temperature does not fall the possibility of empyema. This complication often develops so insidiously and without any event to mark its occurrence that it may readily be overlooked, and this is the more likely to occur, as the changes in the breath sounds and other evidences which suggest consolidation may remain without substantial modification, even when the fluid accumulation within the pleural cavity is considerable in amount. One fact of significance in connection with these residual effusions is the presence of a cough which is essentially paroxysmal in character, comes on after movement, and is unattended by any expectoration. Such a cough following on the ordinary symptoms of acute pneumonia may be regarded as patho.. gnomonic of the presence of fluid, though the volume of fluid may be very small.

Another practical point in connection with pleural effusion when this occurs in the left side is that occasionally it is found that the heart is not displaced. This has been shown to be due to adhesions, the results of a former pleurisy.

$$
{ }_{1} \text { The Lancet, Decembør ž 0, } 1905 .
$$

\section{DIPHTHERITIC HEMIPLEGIA.}

DR. J. D. Rolleston ${ }^{1}$ reports a case of this rare condition. The patient, a boy of six years, was admitted to hospital with faucial diphtheria on November 21, 1904. The severe nature of the case was illustrated by such events as weak and irregular action of the heart, considerable enlargement of the liver and very scanty urine. On December 8 there was observed paralysis of right lower face and of right upper limb, with also some palsy of the right lower limb. There was at the same time motor aphasia. Both knee-jerks were absent, and the plantar response on the right side was of the extensor type, that of the left being normal. On December 10 there was paralysis of accommodation, and on the 23rd paralysis of the muscles of deglutition. Signs of improvement were noted early in the following month, and when the patient left the hospital at the cnd of March his speech had returned, and he could walk fairly well, though there was still some weakness of the right grasp.

Hemiplegia following diphtheria is a very unvsual condition. It differs from the ordinary forms of diphtheritic paralysis in that it is dependent, not on a primary lesion of the nervous apparatus, but on a vascular event-thrombosis, embolism, or hæmorrhagic encephalitis. In all the reported cases the initial faucial attack was severe. Complete recovery is rare; such consequences as contracture, hemichorea, athetosis, and idiocy have been noted.

1 Med. Press and Circ., Dec. 20, 1905.

\section{ACUTE INFECTIVE DIARRHEA OF INFANTS.}

Ir a recent clinical lecture Dr. Batten ${ }^{1}$ deals with this disease on the basis of a special experience while in charge of a special ward set aside for the treatment of such cases at the hospital for sick children, Great Ormond Street.

In the first place, it is to be recognised that in the matter of nursing it is necessary to treat these patients as capable of infecting others by their discharges; to regard the disease as infective in the sense in which enteric fever is infective, and therefore as a disease to be treated with such precautions as enteric fever demands. With this object in view special nurses were appointed to remove soiled linen, and these nurses took no part in the feeding of the infants.

The characteristic features of acute infective diarrhœa in Dr. Batten's estimation are an acute onset attended by frequent vomiting; frequent motions of loose watery consistency, which in the early stage contain neither mucus nor blood, are green in colour and not offensive. The disease produces a condition of collapse; the extremities are cold and cyanosed, the eyes sunken, the skin shrivelled, and the abdomen generally lax and retracted. The pulse is feeble and small, and the temperature raised.

Out of 120 cases admitted for diarrhœa, 30 conformed to this type, and of these 18 died. The treatment advocated is as follows:-

1. Hypodermic injections of strychnia. A most valuable romedy for extreme collapse. Half a minim of the liquor strychninæ B.P. may be given and repeated in half an hour if necessary. Symptoms of poisoning may set in, but are not likely to be grave.

2. Infusion. Subcutaneous infusion of salt solution (one drachm to the pint of water). The infusion should be done by gravity; the needle should be passed well under the skin of the thorax, and the height of the column of water should be some 18 inches. The heat of the water in the glass receiver should be about $150^{\circ} \mathrm{F}$. Four ounces should be infused, and the time occupied may be anything from 20 minutes to an hour.

3. The hot bath, with or without mustard (preferably with), is most valuable for conditions of 